\title{
Can Serum Anti-Mullerian Hormone Level and LH / FSH Ratio Be Predictive of Clinical Pregnancy Through Intracytoplasmic Sperm Injection - Embryo Transfer?
}

\author{
Tugba ELGUN', Meric KARACAN², Asiye IZEM SANDAL ${ }^{3}$, Tulay IREZ1 \\ Istanbul, Turkey
}

\section{ABSTRACT}

OBJECTIVE: To compare clinical pregnancy rate through ICSI-ET between polycystic ovarian syndrome patients and women with normal ovaries (control group). We also investigated whether serum antiMullerian hormone level or LH/FSH ratio may predict clinical pregnancy rate in both groups.

STUDY DESIGN: In this retrospective study, endocrine/clinical parameters and cycle characteristics of women with polycystic ovarian syndrome $(n=32)$ and women with normal ovaries $(n=115)$ aged $<40$ years were evaluated.

RESULTS: Clinical pregnancy rate in polycystic ovarian syndrome group did not differ from that in the control group $(31.3 \%$ vs. $32.2 \%, p>0.05$, respectively). The $\mathrm{LH} / \mathrm{FSH}$ ratio was significantly higher in women who conceived compared to women who did not in the polycystic ovarian syndrome group (0.9 vs. 0.6 , respectively, $p=0.4$ ). The cut-off value of 0.6 in the $\mathrm{LH} / \mathrm{FSH}$ ratio predicted clinical pregnancy with a specificity of $76 \%$ and a sensitivity of $65 \%$ in the polycystic ovarian syndrome group. AntiMullerian hormone was significantly higher in women who conceived compared to women who did not in the control group (4.0 ng/mL vs. $2.1 \mathrm{ng} / \mathrm{mL}$, respectively, $p=0.4)$.

CONCLUSION: Polycystic ovarian syndrome patients have a similar clinical pregnancy rate with women having normal ovaries through ICSI-ET. The LH/FSH ratio assessed prior to ovulation induction was significantly higher in pregnant polycystic ovarian syndrome patients compared to polycystic ovarian syndrome patients who did not conceive. Anti-Mullerian hormone level was significantly higher in pregnant women compared to non-pregnant women with normal ovaries.

Keywords: Polycystic ovary syndrome, Anti-Mullerian hormone, LH/FSH ratio, Intracytoplasmic sperm injection

Gynecol Obstet Reprod Med 2020;26(1):25-30

${ }^{1}$ Biruni University Medical Faculty, Department of Histology and Embryology, Istanbul

2 Yeni Yuzyll University Medical Faculty Department of Obstetrics and Gynecology, Istanbul

${ }^{3}$ Istanbul University Veterinary Faculty, Istanbul

Address of Correspondence: Tulay Irez

Biruni University Medical Faculty Department of Histology \& Embryology 10. Yıl Cad. Protokol Yolu No: 45 34100 Zeytinburnui Istanbul, Turkey ireztulay@yahoo.com

Submitted for Publication: Revised for Publication: 18.01.2019 21.02.2019

13.03.2019

Accepted for Publication:

11-6892, $M K: 0000-0002-7050-3733$

ORCID ID: TE:0000-0002-1311-6892, MK: 0000-0002-76
AIS: 0000-0002-4952-7861, TI: 0000-0001-8272-4931

\begin{tabular}{|c|c|}
\hline \multirow{3}{*}{ 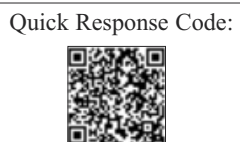 } & Access this article online \\
\hline & $\begin{array}{l}\text { Website: www.gorm.com.tr } \\
\text { e- mail: info@gorm.com.tr }\end{array}$ \\
\hline & DOI:10.21613/GORM.2018.897 \\
\hline
\end{tabular}

How to cite this article: Elgun T. Karacan M. Izem Sandal A. Irez T. Can Serum Anti-Mullerian Hormone Level and LH / FSH Ratio Be Predictive of Clinical Pregnancy Through Intracytoplasmic Sperm Injection - Embryo Transfer? Gynecol Obstet Reprod Med 2020;26(1):25-30

\section{Introduction}

Polycystic ovary syndrome (PCOS) is a common endocrine disorder at reproductive age detected in $6-8 \%$ of women (1-3). PCOS presenting mainly with chronic anovulation and infertility has become a public health problem in recent years due to the risks such as type II diabetes mellitus, dyslipidemia, cardiovascular disease, and endometrial carcinoma. Rotterdam consensus is now considered to be the global standard to define (4). The primary etiology remains unclear but hormonal/metabolic abnormalities namely hyperandrogenemia and insulin resistance often contribute to the underlying pathology.

Anti-Mullerian Hormone (AMH) is a glycoprotein secreted from the granulosa cells of preantral and antral follicles. Although AMH and antral follicle count (AFC) are generally found to be higher in PCOS patients compared to patients with normal ovaries they still have not been included in the diagnostic criteria. AMH levels in women with PCOS are two to three times higher than patients without PCOS (5). 
Various biomarkers have been used to predict pregnancy rates through in vitro fertilization (IVF) cycles such as AMH, AFC, inhibin B, FSH. A positive association between AMH and clinical pregnancy was found in some studies (6-9) whereas others did not confirm it (10). It is also controversial whether AMH levels in women with PCOS provide useful information on the IVF outcome.

Serum LH / FSH ratio used in the diagnosis of PCOS in the past and positively correlated with AFC is not being routinely tested at present (11). On the other hand, there is no study evaluating the importance of this ratio on the prediction of pregnancy in PCOS patients.

In our study, we aimed to compare outcome of intracytoplasmic sperm injection - embryo transfer (ICSI-ET) cycles in PCOS patients and non-PCOS patients less than 40 years old. We also evaluated whether serum AMH level or LH/FSH ratio assessed prior to ovulation induction may predict clinical pregnancy rate in both groups.

\section{Material and Method}

A total of 147 patients who applied for IVF treatment between February 2016 and March 2018 to Ota-Jinemed Hospital were retrospectively analyzed. Of those, 32 patients were diagnosed as having PCOS (PCOS group) according to Revised Rotterdam criteria and 115 patients having normal ovaries with regular menstrual cycles (control group). The inclusion criteria were: women with no systemic disease, age between 25-39 years, body mass index (BMI) $18-30 \mathrm{~kg} / \mathrm{m}^{2}$, fresh cycles and normal or subnormal semen findings in their spouses. Exclusion criteria were based on the following criteria: high basal FSH level $>15,<5$ antral follicles on cycle day 3 and a previous failed attempt. Ethical approval was obtained from the Ethics Committee of Biruni University (27.11.2017, $\# 2017 / 10-8$ ) and the study was conducted in accordance with the Declaration of Helsinki. Consent for the use of the data was obtained from the hospital where patients applied to.

Anti-Mullerian Hormone, FSH, LH, inhibin B, testosterone and DHEAS levels were analyzed on cycle day 3 before initiation of ovulation induction. AMH level was assessed using electrochemiluminescence immunoassay (ECLIA, Roche e170). The FSH and LH levels were measured by VIDAS enzyme-linked fluorescent assay (Biomerieux).

Ovulation induction was initiated on cycle day 3 with 150 IU (Gonal-F, Merck Serono, Switzerland) per day in all patients and adjusted according to ovarian response. A daily administration of $0.25 \mathrm{mg}$ of cetrorelix (Cetrotide; Merck Serono, Switzerland) was introduced in a fixed way on day 6 and continued until the day of recombinant hCG (Ovitrelle, Merck Serono, Switzerland) injection that was administrated at least three follicles reach $17 \mathrm{~mm}$ in diameter. Oocyte pickup (OPU) was performed 35-36 hours later. After the proce- dure, oocytes were placed in Single Step Medium and incubated for 2 hours at $37{ }^{\circ} \mathrm{C}$ under $6 \% \mathrm{CO}_{2}$ and $5 \% \mathrm{O}_{2}$. At the end of 2 hours, oocyte cumulus dissection was performed in the modified human tubular fluid with the aid of hyaluronidase enzyme and metaphase II (MII) oocytes were separated and cultured until ICSI.

\section{Sperm Preparation}

Sperm samples were collected after 3 days of sexual abstinence and evaluated according to the World Health Organization (WHO) laboratory guidelines; sperm concentration, semen volume, sperm motility, and morphology records were recorded (12). As a standard for sperm preparation, in all cases, gradient technique was performed using Isolate Sperm Separation Medium.

\section{Microinjection processing (ICSI)}

Intracytoplasmic sperm injection was performed using an inverted microscope with Hoffman modulation at $37^{\circ} \mathrm{C}$ under X 400 magnification in a preliminarily prepared ICSI dish. Hepes-buffered HTF medium was utilized under mineral oil.

\section{Fertilization and Evaluation of embryos}

High-quality embryos were defined as embryos developed from normal fertilized eggs, with no fragmentation or no more than $10 \%$ fragmentation, in the absence of multinucleation and reaching 7-8 blastomeres $72 \mathrm{~h}$ after the OPU. One embryo in patients $<35$ years or two embryos in those $\geq 35$ years were transferred on the fifth day following OPU. Number of oocytes collected, number of oocytes with germinal vesicle (GV), degenerated oocytes, metaphase II oocytes, number of fertilized oocytes, number of cleaved embryos and clinical pregnancy rates were recorded. Embryo evaluation was performed according to ESHRE, ASRM rules (17). Clinical pregnancy was defined as the presence of fetal heartbeat.

\section{Statistical analysis}

Group characteristics were calculated and compared using the arithmetical means and the standard deviations were for each group as well. The numerical data of the patients were evaluated with Independent T-Test and Kruskal-Wallis test. Receiver Operation Characteristics Curve (ROC) was utilized to detect sensitivity and specificity. A p-value of $<0.05$ was considered to be statistically significant. Statistical analyses were performed using Statistical Package for the Social Sciences (SPSS, IBM, NY, USA) software version 19.0.

\section{Results}

The mean age of men, sperm concentration, percent of motility, and morphology according to Kruger's criteria were similar between the PCOS group and control group (Table I).

The mean age of women between the PCOS group and the control group was similar $(29.8 \pm 5.1$ vs. $31.0 \pm 4.6$ years, respectively). In comparison with the control group; BMI, AMH 
level, LH level, LH/FSH ratio, testosterone level, DHEAS level and AFC in PCOS patients were significantly higher and the dose of gonadotropins used was lower (Table II). Inhibin $\mathrm{B}$, basal FSH, and estradiol level on the day of hCG did not differ significantly between the two groups.

Total number of oocytes collected, oocytes with germinal vesicles, metaphase I oocytes and the degenerated oocytes were significantly higher in the PCOS group as compared to the control group (Table III). The number of cleaved embryos and the number of blastocysts did not differ between the control group and PCOS group (4.4 \pm 2.8 vs. $5.1 \pm 2.3$ and $3.3 \pm 0.4$ vs. $3.6 \pm 0.5$, respectively). Clinical pregnancy rates were similar in the PCOS group and the control group (31.3\% vs. $32.2 \%$, respectively).

$\mathrm{LH} / \mathrm{FSH}$ ratio and pregnancy rate in the polycystic ovary syndrome patients

LH/FSH ratio was significantly higher in pregnant women than that in non-pregnant women in PCOS group (0.9 vs 0.6, respectively, $p=0.4$ ), although AMH levels were not different (Table IV). LH / FSH ratio of 0.68 has a sensitivity of $65 \%$ and a specificity of $76 \%$ to predict clinical pregnancy in the PCOS group (Figure 1).

Table I: Mean age and mean sperm characteristics of men in the control and polycystic ovary syndrome groups

\begin{tabular}{lccc}
\hline Variable & Control Group & PCOS Group & $p$ \\
& $\mathrm{n}=115$ & $\mathrm{n}=32$ & $\mathrm{NS}$ \\
Mean age of men & $35.6 \pm 5.3$ & $34.3 \pm 6.6$ & $\mathrm{NS}$ \\
Sperm concentration $\times 10^{6}$ & $61.1 \pm 47.1$ & $54.4 \pm 54.1$ & $\mathrm{NS}$ \\
Motility (\%) & $30.6 \pm 15.9$ & $29.6 \pm 14.6$ & $\mathrm{NS}$ \\
Morphology (\%) & $3.7 \pm 3.5$ & $3.4 \pm 3.2$ & \\
\hline
\end{tabular}

Note: Results are expressed as mean \pm SD. NS, not significant. PCOS: Polycystic ovary syndrome

Table II: Demographic analysis of patients

\begin{tabular}{|c|c|c|c|}
\hline Variable & $\begin{array}{c}\text { Control Group } \\
n=115\end{array}$ & $\begin{array}{c}\text { PCOS Group } \\
n=32\end{array}$ & $\begin{array}{l}p \\
\text { value }\end{array}$ \\
\hline Age of women (years) & $31.0 \pm 4.6$ & $29.8 \pm 5.1$ & NS \\
\hline Body mass index (BMI) & $24.4 \pm 4.3$ & $28.5 \pm 5.0$ & 0.01 \\
\hline Inhibin B (pg/mL) & $79.6 \pm 49.7$ & $74.8 \pm 47.8$ & NS \\
\hline $\mathrm{AMH}(\mathrm{ng} / \mathrm{dL})$ & $3.1 \pm 2.68$ & $7.5 \pm 3.02$ & 0.002 \\
\hline $\mathrm{FSH}(\mathrm{IU} / \mathrm{mL})$ & $6.5 \pm 2.3$ & $5.6 \pm 2.9$ & NS \\
\hline LH (IU/mL) & $2.9 \pm 1.2$ & $4.7 \pm 1.0$ & 0.02 \\
\hline $\mathrm{LH} / \mathrm{FSH}$ & $0.5 \pm 0.2$ & $0.8 \pm 0.2$ & 0.03 \\
\hline Testosterone (ng/mL) & $25.1 \pm 15.3$ & $42.2 \pm 16.1$ & 0.02 \\
\hline $\operatorname{DHEAS}(\mu \mathrm{g} / \mathrm{dL})$ & $86.2 \pm 30.1$ & $195.1 \pm 25.1$ & 0.001 \\
\hline AFC & $6.98 \pm 3.55$ & $15.28 \pm 6.76$ & 0.001 \\
\hline Total dose of gonadotropins & $2629.1 \pm 1168.5$ & $1748.2 \pm 613.8$ & 0.002 \\
\hline E2 $(p g / m L)$ & $1173.4 \pm 866.8$ & $2264.83 \pm 1794.5$ & NS \\
\hline
\end{tabular}

Note: Results are expressed as mean \pm SD. NS, not significant. PCOS: Polycystic ovary syndrome

Table III: Cycle outcomes of both groups

\begin{tabular}{lcrr}
\hline Variable & Control Group & PCO Group & \multicolumn{1}{c}{$\begin{array}{c}\mathrm{n}=32 \\
\text { value }\end{array}$} \\
\hline No. of oocytes & $8.0 \pm 4.3$ & $11.2 \pm 5.7$ & 0.01 \\
No. of fertilized oocytes & $4.3 \pm 2.6$ & $5.1 \pm 2.9$ & $\mathrm{NS}$ \\
No. of oocytes with germinal vesicle & $0.8 \pm 1.3$ & $2.2 \pm 2.8$ & 0.01 \\
No. of Metaphase I & $0.1 \pm 0.4$ & $0.1 \pm 0.3$ & $\mathrm{NS}$ \\
No. of degenerated oocytes & $0.6 \pm 0.9$ & $1.3 \pm 2.0$ & 0.03 \\
No. of cleaved embryos & $4.4 \pm 2.8$ & $5.1 \pm 2.3$ & $\mathrm{NS}$ \\
No. of blastocyts & $3.3 \pm 0.4$ & $3.6 \pm 0.5$ & $\mathrm{NS}$ \\
Clinical pregnancy rate (\%) & 32.2 & 31.3 & $\mathrm{NS}$ \\
\hline
\end{tabular}

Note: Results are expressed as mean \pm SD. NS. not significant. PCOS: Polycystic ovary syndrome 
Table IV: Serum anti-Mülleriane hormone level and LH/FSH ratio related to clinical pregnancy in polycystic ovary syndrome group

\begin{tabular}{lccc}
\hline & Pregnant & Not pregnant & $p$ \\
$\mathrm{n}=10$ & value & $\mathrm{NS}$ & \\
\hline AMH $(\mathrm{ng} / \mathrm{ml})$ & $9.4 \pm 5.0$ & $6.6 \pm 4.6$ & 0.04 \\
LH/FSH & $0.9 \pm 0.9$ & $0.6 \pm 0.4$ & 0.4 \\
\hline
\end{tabular}

Note: Values are mean $\pm S D$. NS, not significant

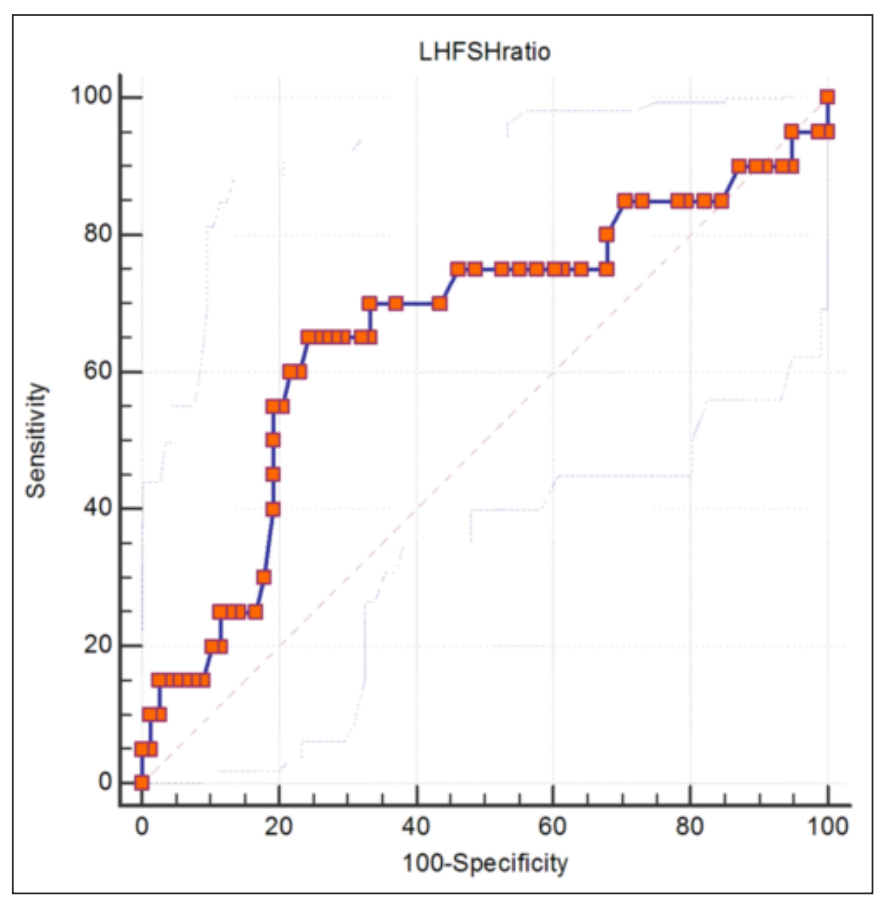

Figure 1: Sensitivity and specificity of $L H / F S H$ ratio in the prediction of pregnancy in the PCOS group. Note: LH / FSH ratio of 0.68 has a sensitivity of $65 \%$ and a specificity of $76 \%$ to predict clinical pregnancy.

Anti-Mullerian Hormone level and pregnancy rate in non-polycystic ovary syndromepatients

Anti-Mullerian Hormone level was significantly higher in pregnant patients than that in non-pregnant patients in the control group (4.0 ng/ml vs. $2.1 \mathrm{ng} / \mathrm{mL}$, respectively, $p=0.4$ ), although LH/FSH ratio did not exhibit any difference (Table V). Serum AMH level of $3.25 \mathrm{ng} / \mathrm{mL}$ has a sensitivity of $76 \%$ and specificity of $69 \%$ to predict clinical pregnancy in the control group (Figure 2).

\section{Discussion}

In this retrospective study, it was demonstrated that PCOS patients and non-PCOS patients less than 40 years had similar

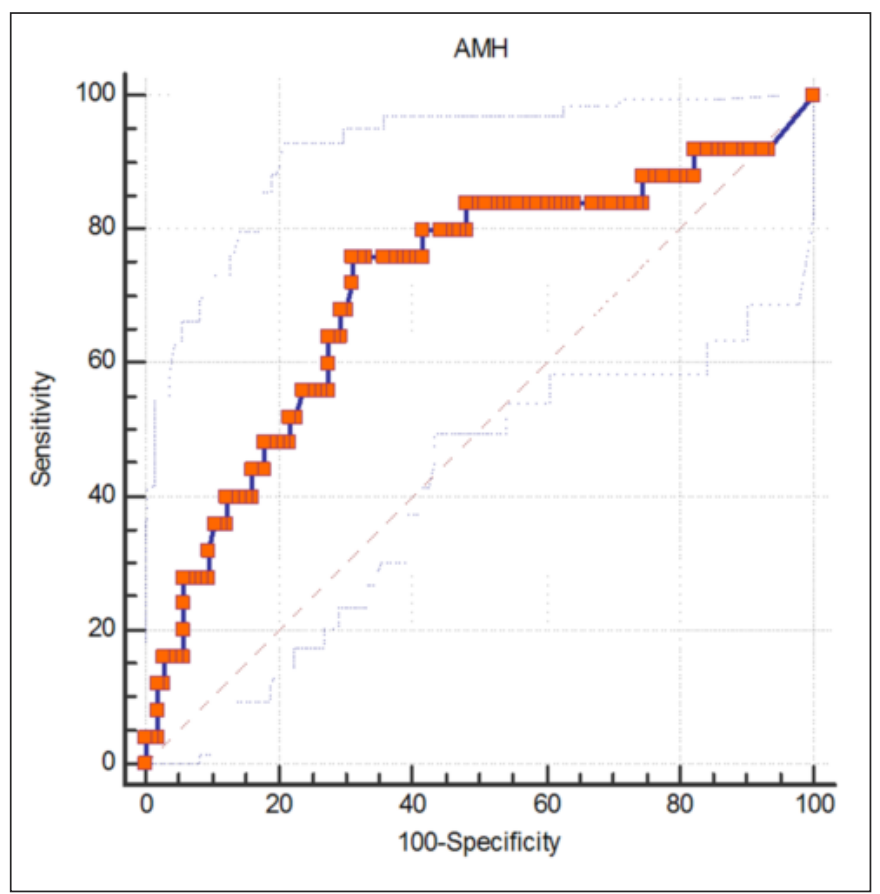

Figure 2: Sensitivity and specificity of AMH for pregnancy prediction in the Control group (patients with normal ovaries).

Note: AMH level of $3.25 \mathrm{ng} / \mathrm{mL}$ predicted clinical pregnancy with a sensitivity of $76 \%$ and a specificity of $69 \%$.

clinical pregnancy rates through ICSI-ET. LH/FSH ratio in PCOS patients and AMH level in non-PCOS patients have predictive values on clinical pregnancy.

Abnormalities in the balance of reproductive hormones result in anovulation, hyperandrogenism in PCOS patients. Yet the effect of hormonal imbalance in these patients on pregnancy rate is still debatable. Women with clinical symptoms of PCOS are known to have adverse outcomes in IVF cycles despite having a good number of follicles, namely compromised oocyte quality, poor quality of embryos and higher miscarriage rates (13). On the contrary, women with morphological features of PCO on sonogram without any of clinical signs of PCOS were reported to have higher number of oocytes, em-

Table V: Serum anti-Mülleriane hormone level and LH/FSH ratio related to clinical pregnancy in the control group

\begin{tabular}{lccc}
\hline & Pregnant & Not pregnant & $p$ \\
& $\mathrm{n}=37$ & $\mathrm{n}=78$ & value \\
\hline $\mathrm{AMH}(\mathrm{ng} / \mathrm{mL})$ & $3.7 \pm 2.1$ & $2.1 \pm 2.9$ & 0.04 \\
$\mathrm{LH} / \mathrm{FSH}$ & $0.7 \pm 0.1$ & $0.5 \pm 0.2$ & $\mathrm{NS}$ \\
\hline
\end{tabular}

Note: Values are mean $\pm S D$. NS, not significant 
bryos and higher cumulative pregnancy rates, but similar miscarriage rates indicating comparable oocyte quality with women having normal ovaries $(13,14)$. In the present study clinical pregnancy rate in PCOS patients was similar to women with normal ovaries.

Anti-Mullerian Hormone level was shown to be a good biomarker for the prediction of clinical pregnancy, with a level less than $0.2 \mathrm{ng} / \mathrm{mL}$ indicating poor prognosis (15). Tal et al. reported that the increased AMH level implied a better response to ovarian stimulation and higher pregnancy rates (16). Hamdine et al. demonstrated that $\mathrm{AMH}$ as a single test had a substantial accuracy in the prediction of ovarian response in GnRH antagonist treatment for IVF (12). Different serum $\mathrm{AMH}$ concentrations have been shown to be closely related to oocyte maturation and quality, embryo development and IVFICSI (17). Basal AMH serum concentration was found to be the strongest predictor of oocyte yield and the level of AMH on the day of hCG injection pertinent to embryo quality (18). The fact that the levels within the cycle and between the cycles are not highly variable makes it a good indicator for ovarian reserve (19). In accordance with previous studies, AMH was found to be a reliable biomarker to predict clinical pregnancy through ICSI-ET in women with normal ovaries in the present study.

Anti-Mullerian Hormone levels were reported to be 2-3 times higher in patients with PCOS than in normal cases (20), which is similar to the finding in the present study. Increased $\mathrm{AMH}$ in serum and especially in follicular fluid in patients with PCOS might impair oocyte maturation, development and embryo quality (21). The number of oocytes was high as well as the number of GV and degenerated oocytes in PCOS patients in the present study.

Anti-Mullerian Hormone level in the follicular fluid was also shown to be strongly correlated with pregnancy rates in the IVF cycle (22). Yilmaz et al. demonstrated that follicular fluid AMH levels predicted pregnancy in women under 35 years of age (23).

Disorders in the function of the hypothalamus-pituitaryovarian axis have been described in PCOS. The amplitude and frequency of the LH pulses and the mean serum LH concentration were found to be increased probably due to the increased GnRH pulse frequency, increased response to $\mathrm{GnRH}$, and high levels of estrogen (24). Mean LH level and LH/FSH ratio were significantly higher in PCOS patients compared to non-PCOS patients in the present study. Moreover, LH/FSH ratio was found to be higher in PCOS patients who conceived compared to those who did not. Serum LH / FSH value in patients with PCOS was reported to be positively correlated with total follicle count and ovarian volume (25).

Higher LH concentrations in PCOS patients could also be attributed to the higher androgen levels, which desensitize the hypothalamus to the negative feedback regulation by progesterone (26). PCOS patients with LH/FSH ratio $>1.5$ had higher basal testosterone, estradiol and AFC and diminished pregnancy rates (27). This may be due to the deleterious effect of LH on folliculogenesis and endometrial receptivity. On the contrary to previous study, LH/FSH ratio was found to be higher in pregnant PCOS compared to non-pregnant PCOS patients.

Zhang et al. reported that serum AMH and inhibin B levels were valuable to predict the ovarian response of PCOS patients (28). In the present study, AMH and inhibin B levels were similar between pregnant and non-pregnant PCOS patients.

The major limitations of the present study are the retrospective design, exclusion of poor prognosis patients and the sample size. The strengths of the study are that all procedures were performed by the same embryologist, high number of markers analyzed prior to ovulation induction and the first clinically meaningful expression of LH/FSH ratio on the prediction of pregnancy. Further studies with a higher sample size are needed to confirm these effects.

\section{Conclusions}

Although having higher number of oocytes retrieved, PCOS patients have a similar clinical pregnancy rate with women having normal ovaries through ICSI-ET. The LH / FSH ratio assessed prior to ovulation induction was significantly higher in pregnant PCOS patients compared to PCOS patients who did not conceive. AMH level was significantly higher in pregnant women compared to non-pregnant women with normal ovarie

\section{:Acknowledgement}

We declare that there is no conflict of interest or funding in this study.

\section{References}

1. Dunaif A, Segal KR, Shelley DR, Green G, Dobrjansky A, Licholai T. Evidence for distinctive and intrinsic defects in insulin action in polycystic ovary syndrome. Diabetes. 1992;41(10):1257-66.

2. Franks S. Polycystic ovary syndrome. N Engl J Med. 1995 28;333(13):853-61.

3. Knochenhauer ES, Key TJ, Kahsar-Miller M, Waggoner W, Boots LR, Azziz R. Prevalence of the polycystic ovary syndrome in unselected black and white women of the southeastern United States: a prospective study. J Clin Endocrinol Metab. 1998;83(9):3078-82.

4. Rotterdam ESHRE/ASRM Sponsored PKOS Consensus Workshop Group. Revised 2003 consensus on diagnostic criteria and long-term health risks related to polycystic ovary syndrome. Fertil Steril 2004 Jan;81(1):19-25. 
5. Wiweko B, Maidarti M, Priangga MD, Shafira N, Fernando D, Sumapraja K, et al. Anti-mullerian hormone as a diagnostic and prognostic tool for PCOS patients. J.Assist Reprod Genet. 2014; 31(10):1311-6.

6. Sahmay S, Guralp O, Aydogan B, Cepni I, Oral E, Irez T. Anti mullerian hormone and polycystic ovary syndrome: assessment of the clinical pregnancy rates in invitro fertilization patients. Gynecol Endocrinol. 2013; 29(5):440-3.

7. Ocal P, Sahmay S, Çetin M, Irez T, Guralp O, Cepni I. Serum anti mullerian hormone and antral follicle count as predictive markers of OHSS in ART cycles. J Assist Reprod Genet. 2011;28(12):1197-203.

8. Irez T, Ocal P, Guralp O, Cetin M, Aydogan B, Sahmay S. Different serum antimullerian hormone concentrations are associated with oocyte quality embryo development parameters and IVF-ICSI outcomes. Arch Gynecol Obstet. 2011;284(5):1295-301.

9. Zhang F, Liu XL, Rong N, Huang XW. Clinical value of serum anti-mullerian hormone and inhibin $\mathrm{B}$ in prediction of ovarian response in patients with polycystic ovary syndrome. J Huazhong Univ Sci Technolog Med Sci. 2017;37(1):70-3.

10. van Rooij IA, Broekmans FJ, Hunault CC, Scheffer GJ, Eijkemans MJ, de Jong FH et al. Use of ovarian reserve tests for the prediction of ongoing pregnancy in couples with unexplained or mild male infertility. Reprod Biomed Online. 2006;12(2):182-90.

11. Chin KV, Seifer DB, Feng B, Lin Y, Shin WC. DNA microarray analysis of the expression profiles of luteinized granulosa cells as a function of ovarian reserve. Fertil Steril. 2002;77(6):1214-8.

12. Hamdine O, Eijkemans MJ, Lentjes EW, Torrance HL, Macklon NS, Fauser BC, et al. Ovarian response prediction in GnRH antagonist treatment for IVF using antiMullerian hormone. Hum Reprod. 2015;30(1):170-8.

13 Nikolaou D, Gilling-Smith C. Early ovarian ageing: Are women with polycystic ovaries protected? Hum Reprod. 2004;19(10):2175-9.

14. Engmann L, Maconochie N, Sladkevicius P, Bekir J, Campbell S, Tan SL. The outcome of in-vitro fertilization treatment in women with sonographic evidence of polycystic ovarian morphology. Hum Reprod. 1999;14(1):16771 .

15. Zebitay AG, Cetin O, Verit FF, Keskin S, Sakar MN, Karahuseyinoglu $\mathrm{S}$, et al. The role of ovarian reserve markers in prediction of clinical pregnancy. J Obstet Gynaecol. 2017;37(4):492-7.

16. Tal R, Seifer DB, Khanimov M, Malter HE, Grazi RV, Leader B. Characterization of women with elevated antimüllerian hormone levels (AMH): correlation of $\mathrm{AMH}$ with polycystic ovarian syndrome phenotypes and assisted reproductive technology outcomes. Am J Obstet Gynecol. 2014;211(1):59.e1-8.
17. Stracquadanio M, Ciotta L, Palumbo MA. Relationship between serum anti-Mullerian hormone and intrafollicular AMH levels in PCOS women. Gynecol Endocrinol. 2018;34(3):223-8.

18. Melado-Vidales L, Fernández-Nistal A, Martínez Fernández V, Verdú Merino V, Bruna Catalán I, Bajo Arenas JM. Anti-Mullerian hormone levels to predict oocyte maturity and embryo quality during controlled ovarian hyperstimulation. Minerva Ginecol. 2017;69 (3):225-32.

19. Ebner T, Sommergruber M, Moser M, Shebl O, SchreierLechner E, Tews G. Basal level of anti-Mullerian hormone is associated with oocyte quality in stimulated cycles. Hum Reprod. 2006;21(3):2022-6.

20. Skałba P, Cygal A, Madej P, Dąbkowska-Huć A, Sikora J, Martirosian G, et al. Is the plasma anti-Mullerian hormone (AMH) level associated with body weight and metabolic, and hormonal disturbances in women with and without polycystic ovary syndrome? Eur J Obstet Gynecol Reprod Biol. 2011;158(2):254-9.

21. Qiao J, Feng HL. Extra- and intra-ovarian factors in polycystic ovary syndrome: impact on oocyte maturation and embryo developmental competence. Hum Reprod Update. 2011;17(1):17-33.

22. Fanchin R, Mendez Lozano DH, Frydman N, Gougeon A, di Clemente N, Frydman R, et al. Anti-Mullerian hormone concentrations in the follicular fluid of the preovulatory follicle are predictive of the implantation potential of the ensuing embryo obtained by in vitro fertilization. J Clin Endocrinol Metab. 2007;92(5):1796-802.

23. Yilmaz T, Tavukcuoglu S, Tasdemir S, Esenkaya S, Irez T. Can follicular fluid anti-Mullerian hormone level be a determinant for pregnancy in women under 35 years of age? Int J Womens Health. 2018;6(1):6-10.

24. Franks S. Polycystic ovary syndrome: a changing perspective. Clin Endocrinol (Oxf). 1989;31(1):87-120.

25. Jayasena CN, Franks S. The management of patients with polycystic ovary syndrome. Nat Rev Endocrinol. 2014;10 (10):624-36.

26. Katsikis I, Karkanaki A, Misichronis G, Delkos D, Kandaraki EA, Panidis D. Phenotypic expression, body mass index and insulin resistance in relation to LH levels in women with polycystic ovary syndrome. Eur J Obstet Gynecol Reprod Biol. 2011;156(2):181-5.

27. Wiser A, Shehata F, Holzer H, Hyman JH, Shalom-Paz E, Son WY, et al. Effect of high LH/FSH ratio on women with polycystic ovary syndrome undergoing in vitro maturation treatment. J Reprod Med. 2013;58(5-6):219-23.

28. Zhang F, Liu XL, Rong N, Huang XW. Clinical value of serum anti-mullerian hormone and inhibin $\mathrm{B}$ in prediction of ovarian response in patients with polycystic ovary syndrome. J Huazhong Univ Sci Technolog Med Sci. 2017; 37(1):70-3. 